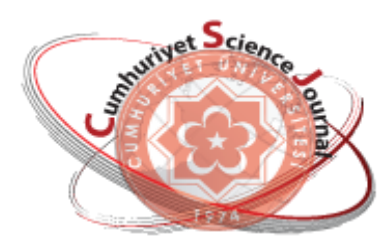

e-ISSN: $2587-246 X$

ISSN: $2587-2680$

\section{Cumburiyet Scionce Journal est}

Cumhuriyet Sci. J., Vol.40-1(2019) 87-101

\title{
On Razzaboni Transformation of Surfaces in Minkowski 3-Space
}

\author{
Melek ERDOĞDU ${ }^{1 *(1 D}$, Mustafa ÖZDEMIR ${ }^{2}$ \\ ${ }^{I}$ Necmettin Erbakan University, Faculty of Science, Department of Mathematics-Computer Science, Konya, \\ TURKEY \\ ${ }^{2}$ Akdeniz University, Faculty of Science, Department of Mathematics, Antalya, TURKEY \\ Received: 19.09.2018; Accepted: 04.01.2019 \\ http://dx.doi.org/10.17776/csj.461375
}

\begin{abstract}
In this paper, we investigate the surfaces generated by binormal motion of Bertrand curves, which is called Razzaboni surface, in Minkowski 3-space. We discussed the geometric properties of these surfaces in $\mathbb{M}^{3}$ with respect to the character of Bertrand geodesics. Then, we define the Razzaboni transformation for a given Razzaboni surface. In other words, we prove that there exists a dual of Razzaboni surface for each case. Finally, we show that Razzaboni transformation maps the surface $\sigma$, which has Bertrand geodesic with constant curvature, to the surface $\sigma^{*}$ whose Bertrand geodesic also has constant curvature with opposite sign.
\end{abstract}

Keywords: Razzaboni surface, Minkowski space.

\section{Minkowski-3 Uzayında Yüzeylerin Razzaboni Dönüşümü Üzerine}

Özet. Bu çalışmada, Minkowski-3 uzayında, Bertrand eğrilerin binormal hareketi ile meydana gelen, Razzaboni yüzeyi adı verilen yüzeyler incelenmiştir. Minkowski-3 uzayındaki bu yüzeylerin geometrik özelliklerini Bertrand geodeziklerin karakterine bağlı olarak tartıştık. Daha sonra, verilen bir Razzaboni yüzeyi için Razzaboni dönüşümünü tanımladık. Diğer bir deyişle, her durum için Razzaboni yüzeyinin bir duali olduğunu ispatladık. Son olarak, Razzaboni dönüşümlerinin; sabit eğrilikli Bertrand geodeziğe sahip $\sigma$ yüzeyini; işareti ters olmak üzere aynı sabit eğrilikli Bertrand geodeziğe sahip $\sigma *$ yüzeyine dönüştürdüğünü gösterdik.

Anahtar Kelimeler: Razzaboni yüzeyi, Minkowski uzayı.

\section{INTRODUCTION}

Bertrand curves are well-studied classical curves in Euclidean space and Lorentzian space [1, 2]. Curves of constant curvature or torsion constitute particular Bertrand curves; and Bertrand curve shares its principal normals with another Bertrand curve, which is called Bertrand mate. The surfaces, which admit geodesic embedding of Bertrand curves, were deeply studied by Amilcare Razzaboni [3]. Then the class of surfaces with Bertrand geodesics came to be called Razzaboni surfaces [4]. In particular, Razzaboni surfaces, which have Bertrand geodesics with constant curvature or torsion, were discussed in earlier work of Razzaboni [5, 6].

The Razzaboni surface can be considered as a surface which is generated by binormal motion of Bertrand curve [4]. The binormal motion of curves with constant curvature and torsion is discussed in [7, 8]. On the other hand, motion of timelike surfaces in timelike geodesic coordinates is examined in the study [9]. 
In this study, we investigate the Razzaboni surfaces in Minkowski 3-space in three different cases. The geometric properties of Razzaboni surfaces in geodesic coordinates are stated for each case. And, we define Razzaboni transformation for a given Razzaboni surface in $\mathbb{M}^{3}$. Then, we examine the curvature and torsion of the Bertrand geodesics of dual Razzaboni surface. Moreover, the Gaussian curvature of Razzaboni surface is given for each case. Finally, the dual Razzaboni surfaces are examined which have geodesics with constant torsion or curvature, respectively.

\section{PRELIMINARIES}

In this part, we give a brief summary of Razzaboni surface in Euclidean 3- Space, [4]. Then, some essential properties of curves in Minkowski 3-space are given to provide the necessary background [10, 11].

Definition 1 Two curves which, at any point, have a common principal normal vector are called Bertrand curves. Moreover, curves for which there exists constants A and B such that

$$
A \kappa+B \tau=1
$$

are also known as Bertrand curves. Here $\kappa$ is the curvature and $\tau$ is the torsion of the curve.

We know that a curve is a geodesic on a surface $\sigma$ if and only if the principal normal of the curve is parallel to the normal $N$ of the surface. This means that if the surface $\sigma$ is spanned by one parameter family of geodesic Bertrand curves $\alpha$ with the same constants $A$ and $B$ then the Bertrand mate $\alpha^{*}$ form a parallel surface $\sigma^{*}$ on which they are likewise geodesics [4].

Let $\sigma=\sigma(u, v)$ be a one parameter family of geodesics with geodesic coordinates. Then the first fundamental form of the surface is of the form

$$
I=d u^{2}+\lambda^{2} d v^{2}
$$

Here, $u$ - parameter curves are unit speed geodesics and $v$ - parameter curves form the orthogonal parallels. Since $\sigma_{u} \perp \sigma_{v}$ and principal normal $n$ of the geodesics is orthogonal to the surface, then the tangent vectors of coordinate line should be of the form

$$
\sigma_{u}=t, \sigma_{v}=\lambda b
$$

where $b$ denotes the binormal vector field of the geodesics. The surface $\sigma$ is generated by the motion of a inextensible curve in binormal direction with the speed $\lambda$. Thus, a Razzaboni surface can be considered as a surface which is generated by binormal motion of Bertrand curve with the same constants $A$ and $B$. The variation of Frenet Frame $\{t, n, b\}$ of the geodesics in $u$ - direction is given by Serret Frenet equations;

$$
\left[\begin{array}{l}
t \\
n \\
b
\end{array}\right]_{u}=\left[\begin{array}{lll}
0 & \kappa & 0 \\
-\kappa & 0 & \tau \\
0 & -\tau & 0
\end{array}\right]\left[\begin{array}{l}
t \\
n \\
b
\end{array}\right]
$$

The variation of $\{t, n, b\}$ in $v$ - direction should be of the form

$$
\left[\begin{array}{l}
t \\
n \\
b
\end{array}\right]_{v}=\left[\begin{array}{lll}
0 & \alpha & \beta \\
-\alpha & 0 & \gamma \\
-\beta & -\gamma & 0
\end{array}\right]\left[\begin{array}{l}
t \\
n \\
b
\end{array}\right]
$$


By using the compatibility condition $\sigma_{u v}=\sigma_{v u}$, we get

$$
\alpha n+\beta b=-\tau \lambda n+\lambda_{u} b .
$$

Thus, the variation of $\{t, n, b\}$ in $v-$ direction becomes

$$
\left[\begin{array}{l}
t \\
n \\
b
\end{array}\right]_{v}=\left[\begin{array}{lll}
0 & -\tau \lambda & \lambda_{u} \\
\tau \lambda & 0 & \gamma \\
-\lambda_{u} & -\gamma & 0
\end{array}\right]\left[\begin{array}{l}
t \\
n \\
b
\end{array}\right]
$$

The compatibility conditions $t_{u v}=t_{v u}, n_{u v}=n_{v u}$ and $b_{u v}=b_{v u}$ gives the following undetermined system

$$
\begin{gathered}
\kappa_{v}=-2 \tau \lambda_{u}-\tau_{u} \lambda, \\
\tau_{v}=\gamma_{u}+\kappa \lambda_{u}, \\
\lambda_{u u}=\tau^{2} \lambda+\kappa \gamma .
\end{gathered}
$$

The above system can be considered as the Gauss-Minardi-Codazzi equations for the surface with geodesic coordinates. If the constraint

$$
A \kappa+B \tau=1
$$

is imposed then the system is well determined and the surface is guaranteed to be a Razzaboni surface [4].

In the case of $A=0$, the geodesics have constant torsion. Choosing $B=\tau=1$ without loss of generality, the system becomes

$$
\begin{gathered}
\kappa_{v}=-2 \lambda_{u}, \\
0=\gamma_{u}+\kappa \lambda_{u}, \\
\lambda_{u u}=\lambda+\kappa \gamma .
\end{gathered}
$$

This integrable system may be regarded as an extension of the sine-Gordon equation;

$$
\Psi_{u v}=\sin \Psi
$$

A single equation is obtained as follows:

$$
\left(\frac{\theta_{v u u}-\theta_{v}}{\theta_{u}}\right)_{u}+\theta_{u} \theta_{v u}=0
$$

by setting $\kappa=\theta_{u}$ and $\lambda=-\frac{\theta_{v}}{2}$.

In the case of $B=0$, the geodesics have constant curvature. Without loss of generality, choosing $A=$ $\kappa=1$, the system becomes

$$
0=-2 \tau \lambda_{u}-\tau_{u} \lambda
$$




$$
\begin{gathered}
\tau_{v}=\gamma_{u}+\lambda_{u}, \\
\lambda_{u u}=\tau^{2} \lambda+\gamma .
\end{gathered}
$$

If we set $\lambda=\frac{1}{\sqrt{\tau}}$, then the system reduces to

$$
\tau_{v}=\left[\left(\frac{1}{\sqrt{\tau}}\right)_{u u}-\tau \sqrt{\tau}+\frac{1}{\sqrt{\tau}}\right]_{u}
$$

which represents an extension of the Dym equation

$$
\tau_{v}=\left(\frac{1}{\sqrt{\tau}}\right)_{u u u}
$$

It can be noted that, the extended Dym equation is generated by binormal motion of an inextensible curve with the speed $\frac{1}{\sqrt{\tau}}$. For further discussions about the Razzaboni surfaces, the readers are referred to [4].

The Minkowski 3-space, denoted by $\mathbb{M}^{3}$, is Euclidean 3-space equipped with Lorentzian inner product

$$
\langle u, v\rangle_{\mathbb{L}}=-u_{1} v_{1}+u_{2} v_{2}+u_{3} v_{3}
$$

where $u=\left(u_{1}, u_{2}, u_{3}\right), v=\left(v_{1}, v_{2}, v_{3}\right) \in \mathbb{M}^{3}$. Lorentzian inner product characterizes the elements $u=$ $\left(u_{1}, u_{2}, u_{3}\right)$ of $\mathbb{M}^{3}$.

if $\langle u, u\rangle_{\mathbb{L}}>0$ or $u=0$ then $u$ is called spacelike,

if $\langle u, u\rangle_{\mathbb{L}}<0$ then $u$ is called timelike,

if $\langle u, u\rangle_{\mathbb{L}}=0$ and $u \neq 0$ then $u$ is called lightlike or null.

The norm of $u=\left(u_{1}, u_{2}, u_{3}\right) \in \mathbb{M}^{3}$ is defined by $\|u\|=\sqrt{|\langle u, u\rangle|}$. Lorentzian vector product of $u=$ $\left(u_{1}, u_{2}, u_{3}\right)$ and $v=\left(v_{1}, v_{2}, v_{3}\right) \in \mathbb{M}^{3}$ is defined by

$$
u \times_{\mathbb{L}} v=\left|\begin{array}{lll}
-e_{1} & e_{2} & e_{3} \\
u_{1} & u_{2} & u_{3} \\
v_{1} & v_{2} & v_{3}
\end{array}\right| .
$$

For details, see [12-14].

A curve $\alpha$ in $\mathbb{M}^{3}$ is called timelike, spacelike or null if and only if tangent vector field $t$ of $\alpha$ is timelike, spacelike or null, respectively. Let $\alpha(s)$ be a unit speed nonlightlike curve in $\mathbb{M}^{3}$, i,e., $\left\langle\alpha^{\prime}, \alpha^{\prime}\right\rangle_{\mathbb{L}}=\varepsilon_{1}=$ \pm 1 . The constant $\varepsilon_{1}$ is called the casual character of $\alpha$. Frenet frame field $\{t, n, b\}$, which is an orthonormal field along $\alpha$, satisfies the Serret-Frenet equation:

$$
\left[\begin{array}{l}
t \\
n \\
b
\end{array}\right]_{s}=\left[\begin{array}{lll}
0 & \varepsilon_{2} \kappa & 0 \\
-\varepsilon_{1} \kappa & 0 & -\varepsilon_{3} \tau \\
0 & \varepsilon_{2} \tau & 0
\end{array}\right]\left[\begin{array}{l}
t \\
n \\
b
\end{array}\right] .
$$


The functions $\kappa \geq 0$ and $\tau$ are called the curvature and torsion, respectively. The constants $\varepsilon_{2}=\langle n, n\rangle_{\mathbb{L}}$ and $\varepsilon_{3}=\langle b, b\rangle_{\mathbb{L}}$ are called the second casual character and third casual character of $\alpha$, respectively [10$12]$.

\section{RAZZABONI TRANSFORMATION OF SURFACES IN MINKOWSKI 3-SPACE}

In this part, Razzaboni surfaces in Minkowski 3-space are investigated in three different cases.

\section{Case 1: Geodesic Bertrand curves of Razzaboni surface have timelike principal normal.}

Let $\sigma=\sigma(u, v)$ be a one parameter family of geodesic Bertrand curve with timelike principal normals in $\mathbb{M}^{3}$. And let $u$ and $v$ be the geodesic coordinates of the Razzaboni surface $\sigma$. Then the first fundamental form of the surface is of the form

$$
I=d u^{2}+\lambda^{2} d v^{2}
$$

Here $u$ - parameter curves are unit speed spacelike Bertrand geodesics and $v$ - parameter curves form orthogonal spacelike parallels. Since $\left\langle\sigma_{u}, \sigma_{v}\right\rangle_{\mathbb{L}}=0$ and the principal normal $n$ of geodesics are normal to the surface, then the tangent vectors of coordinate curves are given as

$$
\sigma_{u}=t \text { and } \sigma_{v}=\lambda b
$$

where $b$ denotes the binormal vector field of geodesics. The variation of the Frenet Frame $\{t, n, b$,$\} of$ Bertrand geodesics in $u$ - direction is obtained as follows:

$$
\left[\begin{array}{l}
t \\
n \\
b
\end{array}\right]_{u}=\left[\begin{array}{lll}
0 & -\kappa & 0 \\
-\kappa & 0 & -\tau \\
0 & -\tau & 0
\end{array}\right]\left[\begin{array}{l}
t \\
n \\
b
\end{array}\right]
$$

by setting $\left(\varepsilon_{1}, \varepsilon_{2}, \varepsilon_{3}\right)=(1,-1,1)$ in Equation 1 . The variation of $\{t, n, b\}$ in $v$ - direction must be in the following form:

$$
\left[\begin{array}{l}
t \\
n \\
b
\end{array}\right]_{v}=\left[\begin{array}{lll}
0 & \alpha & \beta \\
\alpha & 0 & \gamma \\
-\beta & \gamma & 0
\end{array}\right]\left[\begin{array}{l}
t \\
n \\
b
\end{array}\right]
$$

The compatibility condition $\sigma_{u v}=\sigma_{v u}$ implies that

$$
\alpha n+\beta b=(-\lambda \tau) n+\lambda_{u} b
$$

So, we get $\alpha=-\lambda \tau$ and $\beta=\lambda_{u}$. Then the variation of $\{t, n, b\}$ in $v$ - direction becomes

$$
\left[\begin{array}{l}
t \\
n \\
b
\end{array}\right]_{v}=\left[\begin{array}{lll}
0 & -\lambda \tau & \lambda_{u} \\
-\lambda \tau & 0 & \gamma \\
-\lambda_{u} & \gamma & 0
\end{array}\right]\left[\begin{array}{l}
t \\
n \\
b
\end{array}\right] .
$$

On the other hand, the compatibility condition $t_{u v}=t_{v u}$ implies that

$$
\begin{gathered}
t_{u v}=(-\kappa n)_{v}=(\lambda \kappa \tau) t+\left(-\kappa_{v}\right) n+(-\kappa \gamma) b, \\
t_{v u}=\left(-\lambda \tau n+\lambda_{u} b\right)_{u}=(\lambda \kappa \tau) t+\left(-\lambda \tau_{u}-2 \lambda_{u} \tau\right) n+(-\kappa \gamma) b .
\end{gathered}
$$


So we get

$$
\kappa_{v}=\lambda \tau_{u}+2 \lambda_{u} \tau
$$

And by the compatibility condition $n_{u v}=n_{v u}$, we obtain

$$
\begin{gathered}
n_{u v}=(-\kappa t-\tau b)_{v}=\left(-\kappa_{v}+\lambda_{u} \tau\right) t+(\kappa \lambda \tau-\tau \gamma) n+\left(-\lambda_{u} \kappa-\tau_{v}\right) b, \\
n_{v u}=(-\lambda \tau t+\gamma b)_{u}=\left(-\lambda_{u} \tau-\lambda \tau_{u}\right) t+(\kappa \lambda \tau-\tau \gamma) n+\left(\gamma_{u}\right) b .
\end{gathered}
$$

Thus, we get

$$
\tau_{v}=-\lambda_{u} \kappa-\gamma_{u}
$$

Finally, the compatibility condition $b_{u v}=b_{v u}$ gives that

$$
\begin{gathered}
b_{u v}=(-\tau n)_{v}=\left(\lambda \tau^{2}\right) t+\left(-\tau_{v}\right) n+(-\tau \gamma) b, \\
b_{v u}=\left(-\lambda_{u} t+\gamma n\right)_{u}=\left(-\lambda_{u u}-\kappa \gamma\right) t+\left(\lambda_{u} \kappa+\gamma_{u}\right) n+(-\tau \gamma) b .
\end{gathered}
$$

Then, we get

$$
\lambda_{u u}=-\lambda \tau^{2}-\kappa \gamma
$$

The system

$$
\begin{aligned}
& \kappa_{v}=\lambda \tau_{u}+2 \lambda_{u} \tau, \\
& \tau_{v}=-\lambda_{u} \kappa-\gamma_{u}, \\
& \lambda_{u u}=-\lambda \tau^{2}-\kappa \gamma
\end{aligned}
$$

may be regarded as the Gauss-Minardi-Codazzi equations for the surface. If the constraint

$$
A \kappa+B \tau=1
$$

is imposed then the system is well determined and the surface $\sigma$ is guaranteed to be a Razzaboni surface.

Now, let's suppose that $\varphi$ is a transformation between two Razzaboni surfaces in $\mathbb{M}^{3}$ defined as $\sigma^{*}=$ $\varphi(\sigma)$. And let $\left\{t^{*}, n^{*}, b^{*}\right\}$ be the Frenet Frame of Bertrand geodesics of Razzaboni surface $\sigma^{*}$. Then the $u^{*}$ - parameter curves are unit speed spacelike Bertrand geodesics and $v^{*}$ - parameter curves form orthogonal spacelike parallels. If the following properties are satisfied

i) $\left|\sigma-\sigma^{*}\right|=$ constant;

ii) $\sigma-\sigma^{*} \perp b$;

iii) $\sigma-\sigma^{*} \perp b^{*}$;

iv) $\left\langle b, b^{*}\right\rangle_{\mathbb{L}}=$ constant, 
then $\varphi$ is called a Razzaboni transformation and the surface $\sigma^{*}$ is called dual Razzaboni surface of $\sigma$. By the first property, the distance between corresponding points of the surfaces is constant. So, we can write

$$
\sigma^{*}(u, v)=\varphi(\sigma(u, v))=\sigma(u, v)+A n(u, v)
$$

Since the geodesics of the Razzaboni surfaces $\sigma$ and $\sigma^{*}$ are Bertrand mates, we have $n=n^{*}$. Also we get

$$
\sigma_{u}^{*}=\sigma_{u}+A n_{u}=(1-A \kappa) t-A \tau b=\tau(B t-A b) .
$$

That is

$$
t^{*}=\frac{1}{\sqrt{A^{2}+B^{2}}}(B t-A b)
$$

Since $b^{*}=-t^{*} \times_{\mathbb{L}} n^{*}$, we obtain

$$
b^{*}=\frac{1}{\sqrt{A^{2}+B^{2}}}(A t+B b)
$$

And

$$
d u^{*}=\sqrt{A^{2}+B^{2}} \tau d u .
$$

Then, we have

$$
\begin{gathered}
\left\langle\sigma^{*}-\sigma, b\right\rangle_{\mathbb{L}}=\langle A n, b\rangle_{\mathbb{L}}=0, \\
\left\langle\sigma^{*}-\sigma, b^{*}\right\rangle_{\mathbb{L}}=\frac{1}{\sqrt{A^{2}+B^{2}}}\langle A n, A t+B b\rangle_{\mathbb{L}}=0, \\
\left\langle b, b^{*}\right\rangle_{\mathbb{L}}=\frac{1}{\sqrt{A^{2}+B^{2}}}\langle b, A t+B b\rangle_{\mathbb{L}}=\frac{B}{\sqrt{A^{2}+B^{2}}} .
\end{gathered}
$$

Thus, the properties ii), iii) and iv) are also satisfied which means that $\varphi: \sigma \rightarrow \sigma^{*}$ is a Razzaboni transformation. On the other hand, we have

$$
t_{u^{*}}^{*}=\frac{B}{\left(A^{2}+B^{2}\right) \tau} t_{u}-\frac{A}{\left(A^{2}+B^{2}\right) \tau} b_{u}=\frac{-\kappa B+A \tau}{\left(A^{2}+B^{2}\right) \tau} n .
$$

Then, the curvature of Bertrand geodesic of dual Razzaboni surface is found as

$$
\kappa^{*}=\left\langle t_{u^{*}}^{*}, n^{*}\right\rangle_{\mathbb{L}}=\frac{-\kappa B+A \tau}{\left(A^{2}+B^{2}\right) \tau}\langle n, n\rangle_{\mathbb{L}}=\frac{B \kappa-A \tau}{\left(A^{2}+B^{2}\right) \tau} .
$$

Moreover, we have

$$
b_{u^{*}}^{*}=\frac{A}{\left(A^{2}+B^{2}\right) \tau} t_{u}+\frac{B}{\left(A^{2}+B^{2}\right) \tau} b_{u}=\frac{-A \kappa-B \tau}{\left(A^{2}+B^{2}\right) \tau} n=\frac{-1}{\left(A^{2}+B^{2}\right) \tau} n .
$$

Then, we get

$$
\tau^{*}=\left\langle b_{u^{*}}^{*}, n^{*}\right\rangle_{\mathbb{L}}=\frac{-1}{\left(A^{2}+B^{2}\right) \tau}\langle n, n\rangle_{\mathbb{L}}=\frac{1}{\left(A^{2}+B^{2}\right) \tau}
$$


Thus, we have proved the following theorem.

Theorem 1. Let $\sigma=\sigma(u, v)$ be Razzaboni surface with geodesic Bertrand curve having timelike principal normal. Then, the curvature and torsion of Bertrand geodesic of dual Razzaboni surface $\sigma^{*}$ are found as

$$
\begin{aligned}
\kappa^{*} & =\frac{B \kappa-A \tau}{\left(A^{2}+B^{2}\right) \tau}, \\
\tau^{*} & =\frac{1}{\left(A^{2}+B^{2}\right) \tau},
\end{aligned}
$$

respectively.

\section{Case 2: Geodesic Bertrand curves of the Razzaboni surface have timelike binormals.}

Let $\sigma=\sigma(u, v)$ be a one parameter family of geodesic Bertrand curve with timelike binormals in $\mathbb{M}^{3}$. And let $u$ and $v$ be the geodesic coordinates of the Razzaboni surface $\sigma$. Then the first fundamental form of the surface is of the form

$$
I=d u^{2}-\lambda^{2} d v^{2}
$$

Here the curves $u$ - parameter curves are unit speed spacelike Bertrand geodesics and $v$ - parameter curves form orthogonal timelike parallels. Since $\left\langle\sigma_{u}, \sigma_{v}\right\rangle_{\mathbb{L}}=0$ and the principal normal $n$ of geodesics are normal to the surface, then the tangent vectors of coordinate curves are given as

$$
\sigma_{u}=t \text { and } \sigma_{v}=\lambda b
$$

where $b$ denotes the binormal vector field of geodesics. The variation of the Frenet Frame $\{t, n, b$,$\} of$ Bertrand geodesics in $u$ - direction is obtained as follows:

$$
\left[\begin{array}{l}
t \\
n \\
b
\end{array}\right]_{u}=\left[\begin{array}{lll}
0 & \kappa & 0 \\
-\kappa & 0 & \tau \\
0 & \tau & 0
\end{array}\right]\left[\begin{array}{l}
t \\
n \\
b
\end{array}\right]
$$

by setting $\left(\varepsilon_{1}, \varepsilon_{2}, \varepsilon_{3}\right)=(1,1,-1)$ in Equation 1 . The variation of $\{t, n, b\}$ in $v$ - direction must be in the following form:

$$
\left[\begin{array}{l}
t \\
n \\
b
\end{array}\right]_{v}=\left[\begin{array}{lll}
0 & \alpha & \beta \\
-\alpha & 0 & \gamma \\
\beta & \gamma & 0
\end{array}\right]\left[\begin{array}{l}
t \\
n \\
b
\end{array}\right]
$$

The compatibility condition $\sigma_{u v}=\sigma_{v u}$ implies that

$$
\alpha n+\beta b=(\lambda \tau) n+\lambda_{u} b
$$

So, we get $\alpha=\lambda \tau$ and $\beta=\lambda_{u}$. Then the variation of $\{t, n, b\}$ in $v$ - direction becomes

$$
\left[\begin{array}{l}
t \\
n \\
b
\end{array}\right]_{v}=\left[\begin{array}{lll}
0 & \lambda \tau & \lambda_{u} \\
-\lambda \tau & 0 & \gamma \\
\lambda_{u} & \gamma & 0
\end{array}\right]\left[\begin{array}{l}
t \\
n \\
b
\end{array}\right] .
$$


On the other hand, the compatibility condition $t_{u v}=t_{v u}$ implies that

$$
\begin{gathered}
t_{u v}=(\kappa n)_{v}=(-\lambda \kappa \tau) t+\left(\kappa_{v}\right) n+(\kappa \gamma) b, \\
t_{v u}=\left(\lambda \tau n+\lambda_{u} b\right)_{u}=(-\lambda \kappa \tau) t+\left(\lambda \tau_{u}+2 \lambda_{u} \tau\right) n+(\kappa \gamma) b .
\end{gathered}
$$

So we get

$$
\kappa_{v}=\lambda \tau_{u}+2 \lambda_{u} \tau
$$

And by the compatibility condition $n_{u v}=n_{v u}$, we obtain

$$
\begin{gathered}
n_{u v}=(-\kappa t+\tau b)_{v}=\left(-\kappa_{v}+\lambda_{u} \tau\right) t+(-\kappa \lambda \tau+\tau \gamma) n+\left(-\lambda_{u} \kappa+\tau_{v}\right) b \\
n_{v u}=(-\lambda \tau t+\gamma b)_{u}=\left(-\lambda_{u} \tau-\lambda \tau_{u}\right) t+(-\kappa \lambda \tau+\tau \gamma) n+\left(\gamma_{u}\right) b .
\end{gathered}
$$

Thus, we get

$$
\tau_{v}=\lambda_{u} \kappa+\gamma_{u}
$$

Finally, the compatibility condition $b_{u v}=b_{v u}$ gives that

$$
\begin{gathered}
b_{u v}=(\tau n)_{v}=\left(-\lambda \tau^{2}\right) t+\left(\tau_{v}\right) n+(\tau \gamma) b \\
b_{v u}=\left(\lambda_{u} t+\gamma n\right)_{u}=\left(\lambda_{u u}-\kappa \gamma\right) t+\left(\lambda_{u} \kappa+\gamma_{u}\right) n+(\tau \gamma) b .
\end{gathered}
$$

Then, we get

$$
\lambda_{u u}=-\lambda \tau^{2}+\kappa \gamma
$$

The system

$$
\begin{gathered}
\kappa_{v}=\lambda \tau_{u}+2 \lambda_{u} \tau, \\
\tau_{v}=\lambda_{u} \kappa+\gamma_{u}, \\
\lambda_{u u}=-\lambda \tau^{2}+\kappa \gamma
\end{gathered}
$$

can be considered as the Gauss-Minardi-Codazzi equations for the surface. By the constraint

$$
A \kappa+B \tau=1
$$

the system is well determined and the surface $\sigma$ is guaranteed to be a Razzaboni surface.

Similar to case 1 , we can define Razzaboni transformation $\varphi: \sigma \rightarrow \sigma^{*}$ as

$$
\sigma^{*}(u, v)=\sigma(u, v)+A n(u, v)
$$

It is easily seen that the transformation $\varphi$ satisfies all properties of Razzaboni transformation. Here $u^{*}$ and $v^{*}$ are the geodesic coordinates of dual Razzaboni surface $\sigma^{*}$. And let $\left\{t^{*}, n^{*}, b^{*}\right\}$ be the Frenet Frame of Bertrand geodesics of dual Razzaboni surface $\sigma^{*}$. Then the $u^{*}-$ parameter curves are unit speed 
spacelike Bertrand geodesics and $v^{*}-$ parameter curves forms orthogonal timelike parallels. Since the geodesics of the Razzaboni surface and its dual are Bertrand mates, we have $n=n^{*}$. Also we have

$$
\sigma_{u}^{*}=\sigma_{u}+A n_{u}=(1-A \kappa) t+A \tau b=\tau(B t+A b)
$$

That is

$$
t^{*}=\frac{B t+A b}{\sqrt{B^{2}-A^{2}}}
$$

Since $b^{*}=-t^{*} \times_{\mathbb{L}} n^{*}$, we obtain

$$
b^{*}=\frac{-A t+B b}{\sqrt{B^{2}-A^{2}}}
$$

And

$$
d u^{*}=\sqrt{B^{2}-A^{2}} \tau d u
$$

Moreover, we have

$$
\begin{gathered}
t_{u^{*}}^{*}=\frac{B}{\left(B^{2}-A^{2}\right) \tau} t_{u}+\frac{A}{\left(B^{2}-A^{2}\right) \tau} b_{u}=\frac{\kappa B+A \tau}{\left(B^{2}-A^{2}\right) \tau} n, \\
\kappa^{*}=\left\langle t_{u^{*}}^{*}, n^{*}\right\rangle_{\mathbb{L}}=\frac{\kappa B+A \tau}{\left(B^{2}-A^{2}\right) \tau}\langle n, n\rangle_{\mathbb{L}}=\frac{\kappa B+A \tau}{\left(B^{2}-A^{2}\right) \tau} \\
b_{u^{*}}^{*}=-\frac{A}{\left(B^{2}-A^{2}\right) \tau} t_{u}+\frac{B}{\left(B^{2}-A^{2}\right) \tau} b_{u}=\frac{-A \kappa+B \tau}{\left(B^{2}-A^{2}\right) \tau} n, \\
\tau^{*}=\left\langle b_{u^{*}}^{*}, n^{*}\right\rangle_{\mathbb{L}}=\frac{-A \kappa+B \tau}{\left(B^{2}-A^{2}\right) \tau}\langle n, n\rangle_{\mathbb{L}}=\frac{-A \kappa+B \tau}{\left(B^{2}-A^{2}\right) \tau} .
\end{gathered}
$$

Theorem 2. Let $\sigma=\sigma(u, v)$ be Razzaboni surface with geodesic Bertrand curve having timelike binormal. Then, the curvature and torsion of Bertrand geodesic of dual Razzaboni surface $\sigma^{*}$ are found as

$$
\begin{aligned}
& \kappa^{*}=\frac{\kappa B+A \tau}{\left(B^{2}-A^{2}\right) \tau}, \\
& \tau^{*}=\frac{-A \kappa+B \tau}{\left(B^{2}-A^{2}\right) \tau},
\end{aligned}
$$

respectively.

\section{Case 3: Geodesic Bertrand curves of the Razzaboni surface are timelike.}


Let $\sigma=\sigma(u, v)$ be a one parameter family of timelike geodesic Bertrand curve in $\mathbb{M}^{3}$. And let $u$ and $v$ be the geodesic coordinates of the Razzaboni surface $\sigma$. Then the first fundamental form of the surface is of the form

$$
I=-d u^{2}+\lambda^{2} d v^{2}
$$

Here the $u$ - parameter curves are unit speed timelike Bertrand geodesics and $v$ - parameter curves form orthogonal spacelike parallels. Since $\left\langle\sigma_{u}, \sigma_{v}\right\rangle_{\mathbb{L}}=0$ and the principal normal $n$ of geodesics are normal to the surface, then the tangent vectors of coordinate curves are given as

$$
\sigma_{u}=t \quad \text { and } \quad \sigma_{v}=\lambda b
$$

where $b$ denotes the binormal vector field of geodesics. The variation of the Frenet Frame $\{t, n, b$,$\} of$ Bertrand geodesics in $u-$ direction is obtained as follows:

$$
\left[\begin{array}{l}
t \\
n \\
b
\end{array}\right]_{u}=\left[\begin{array}{lll}
0 & \kappa & 0 \\
\kappa & 0 & -\tau \\
0 & \tau & 0
\end{array}\right]\left[\begin{array}{l}
t \\
n \\
b
\end{array}\right]
$$

by setting $\left(\varepsilon_{1}, \varepsilon_{2}, \varepsilon_{3}\right)=(-1,1,1)$ in Equation 1 . The variation of $\{t, n, b\}$ in $v$ - direction must be in the following form:

$$
\left[\begin{array}{l}
t \\
n \\
b
\end{array}\right]_{v}=\left[\begin{array}{lll}
0 & \alpha & \beta \\
\alpha & 0 & \gamma \\
\beta & -\gamma & 0
\end{array}\right]\left[\begin{array}{l}
t \\
n \\
b
\end{array}\right]
$$

The compatibility condition $\sigma_{u v}=\sigma_{v u}$ implies that

$$
\alpha n+\beta b=(\lambda \tau) n+\lambda_{u} b
$$

So, we get $\alpha=\lambda \tau$ and $\beta=\lambda_{u}$. Then the variation of $\{t, n, b\}$ in $v-$ direction becomes

$$
\left[\begin{array}{l}
t \\
n \\
b
\end{array}\right]_{v}=\left[\begin{array}{lll}
0 & \lambda \tau & \lambda_{u} \\
\lambda \tau & 0 & \gamma \\
\lambda_{u} & -\gamma & 0
\end{array}\right]\left[\begin{array}{l}
t \\
n \\
b
\end{array}\right]
$$

On the other hand, the compatibility condition $t_{u v}=t_{v u}$ implies that

$$
\begin{gathered}
t_{u v}=(\kappa n)_{v}=(\lambda \kappa \tau) t+\left(\kappa_{v}\right) n+(\kappa \gamma) b \\
t_{v u}=\left(\lambda \tau n+\lambda_{u} b\right)_{u}=(\lambda \kappa \tau) t+\left(\lambda \tau_{u}+2 \lambda_{u} \tau\right) n+(\kappa \gamma) b .
\end{gathered}
$$

So we get

$$
\kappa_{v}=\lambda \tau_{u}+2 \lambda_{u} \tau
$$

And by the compatibility condition $n_{u v}=n_{v u}$, we obtain

$$
\begin{gathered}
n_{u v}=(\kappa t-\tau b)_{v}=\left(\kappa_{v}-\lambda_{u} \tau\right) t+(\kappa \lambda \tau+\tau \gamma) n+\left(\lambda_{u} \kappa-\tau_{v}\right) b, \\
n_{v u}=(\lambda \tau t+\gamma b)_{u}=\left(\lambda_{u} \tau+\lambda \tau_{u}\right) t+(\kappa \lambda \tau+\tau \gamma) n+\left(\gamma_{u}\right) b .
\end{gathered}
$$


Thus, we get

$$
\tau_{v}=\lambda_{u} \kappa-\gamma_{u}
$$

Finally, the compatibility condition $b_{u v}=b_{v u}$ gives that

$$
\begin{gathered}
b_{u v}=(\tau n)_{v}=\left(\lambda \tau^{2}\right) t+\left(\tau_{v}\right) n+(\tau \gamma) b \\
b_{v u}=\left(\lambda_{u} t-\gamma n\right)_{u}=\left(\lambda_{u u}-\kappa \gamma\right) t+\left(\lambda_{u} \kappa-\gamma_{u}\right) n+(\tau \gamma) b .
\end{gathered}
$$

Then, we get

$$
\lambda_{u u}=\lambda \tau^{2}+\kappa \gamma
$$

The system

$$
\begin{gathered}
\kappa_{v}=\lambda \tau_{u}+2 \lambda_{u} \tau, \\
\tau_{v}=\lambda_{u} \kappa-\gamma_{u}, \\
\lambda_{u u}=\lambda \tau^{2}+\kappa \gamma
\end{gathered}
$$

may be regarded as the Gauss-Minardi-Codazzi equations for the surface. If the constraint

$$
A \kappa+B \tau=1
$$

is imposed then the system is well determined and the surface $\sigma$ is guaranteed to be a Razzaboni surface.

Again, we define the Razzaboni transformation $\varphi: \sigma \rightarrow \sigma^{*}$ as follows;

$$
\sigma^{*}(u, v)=\sigma(u, v)+A n(u, v)
$$

Let $u^{*}$ and $v^{*}$ be the geodesic coordinates of dual Razzaboni surface $\sigma^{*}$. And let $\left\{t^{*}, n^{*}, b^{*}\right\}$ be the Frenet Frame of Bertrand geodesics of dual Razzaboni surface $\sigma^{*}$. Then the $u^{*}-$ parameter curves are unit speed timelike Bertrand geodesics and $v^{*}$ - parameter curves form orthogonal spacelike parallels. Since the geodesics of the Razzaboni surface and its dual are Bertrand mates, we have $n=n^{*}$. Also we have

$$
\sigma_{u}^{*}=\sigma_{u}-A n_{u}=(1-A \kappa) t+A \tau b=\tau(B t+A b) .
$$

That is

$$
t^{*}=\frac{1}{\sqrt{B^{2}-A^{2}}}(B t+A b)
$$

Since $b^{*}=-t^{*} \times_{\mathbb{L}} n^{*}$, we obtain

$$
b^{*}=\frac{1}{\sqrt{B^{2}-A^{2}}}(-A t+B b)
$$


And

$$
d u^{*}=\sqrt{B^{2}-A^{2}} \tau d u .
$$

On the other hand, we have

$$
\begin{gathered}
t_{u^{*}}^{*}=\frac{B}{\left(B^{2}-A^{2}\right) \tau} t_{u}+\frac{A}{\left(B^{2}-A^{2}\right) \tau} b_{u}=\frac{B \kappa+A \tau}{\left(B^{2}-A^{2}\right) \tau} n, \\
\kappa^{*}=\left\langle t_{u^{*}}^{*}, n^{*}\right\rangle_{\mathbb{L}}=\frac{B \kappa+A \tau}{\left(B^{2}-A^{2}\right) \tau}\langle n, n\rangle_{\mathbb{L}}=\frac{B \kappa+A \tau}{\left(B^{2}-A^{2}\right) \tau}, \\
b_{u^{*}}^{*}=\frac{-A}{\left(B^{2}-A^{2}\right) \tau} t_{u}+\frac{B}{\left(B^{2}-A^{2}\right) \tau} b_{u}=\frac{-A \kappa+B \tau}{\left(B^{2}-A^{2}\right) \tau} n, \\
\tau^{*}=\left\langle b_{u^{*}}^{*}, n^{*}\right\rangle_{\mathbb{L}}=\frac{-A \kappa+B \tau}{\left(B^{2}-A^{2}\right) \tau}\langle n, n\rangle_{\mathbb{L}}=\frac{-A \kappa+B \tau}{\left(B^{2}-A^{2}\right) \tau} .
\end{gathered}
$$

Theorem 3. Let $\sigma=\sigma(u, v)$ be Razzaboni surface with timelike geodesic Bertrand curve. Then, the curvature and torsion of Bertrand geodesic of dual Razzaboni surface $\sigma^{*}$ are found as

$$
\begin{aligned}
& \kappa^{*}=\frac{B \kappa+A \tau}{\left(B^{2}-A^{2}\right) \tau}, \\
& \tau^{*}=\frac{-A \kappa+B \tau}{\left(B^{2}-A^{2}\right) \tau},
\end{aligned}
$$

respectively.

\section{CONCLUSION}

In the first case, we obtain that the solutions $\kappa, \tau, \lambda$ and $\gamma$ of the system

$$
\begin{gathered}
\kappa_{v}=\lambda \tau_{u}+2 \lambda_{u} \tau, \\
\tau_{v}=-\lambda_{u} \kappa-\gamma_{u}, \\
\lambda_{u u}=-\lambda \tau^{2}-\kappa \gamma, \\
A \kappa+B \tau=1
\end{gathered}
$$

constitute the spacelike Razzaboni surfaces in $\mathbb{M}^{3}$. In the second case we obtain that the solutions $\kappa, \tau, \lambda$ and $\gamma$ of the system

$$
\begin{gathered}
\kappa_{v}=\lambda \tau_{u}+2 \lambda_{u} \tau, \\
\tau_{v}=\lambda_{u} \kappa+\gamma_{u}, \\
\lambda_{u u}=-\lambda \tau^{2}+\kappa \gamma \\
A \kappa+B \tau=1
\end{gathered}
$$


constitutes the timelike Razzaboni surfaces with timelike $v$ - parameter curves in $\mathbb{M}^{3}$.

For first and second cases, the second fundamental form of the surface $\sigma$ is of the form

$$
I I=-\kappa d u^{2}-2 \lambda \tau d u d v+\frac{\lambda}{\kappa}\left(-\lambda_{u u}-\lambda \tau^{2}\right) d v^{2} .
$$

Then the Gaussian curvature of the surface is obtained as

$$
K=-\frac{\lambda_{u u}}{\lambda}
$$

In the last case, we obtain that the solutions $\kappa, \tau, \lambda$ and $\gamma$ of the system

$$
\begin{gathered}
\kappa_{v}=\lambda \tau_{u}+2 \lambda_{u} \tau, \\
\tau_{v}=\lambda_{u} \kappa-\gamma_{u}, \\
\lambda_{u u}=\lambda \tau^{2}+\kappa \gamma \\
A \kappa+B \tau=1
\end{gathered}
$$

constitute the timelike Razzaboni surfaces with timelike $u$ - parameter curves in $\mathbb{M}^{3}$. In this case, the second fundamental form of the surface is of the form

$$
I I=-\kappa d u^{2}-2 \lambda \tau d u d v+\frac{\lambda}{\kappa}\left(-\lambda_{u u}+\lambda \tau^{2}\right) d v^{2} .
$$

Then Gaussian curvature of the surface is obtained as

$$
K=\frac{\lambda_{u u}}{\lambda}
$$

In the case of $A=0$, which means that Bertrand geodesics of the surface $\sigma$ must have constant torsion

$$
\tau=\frac{1}{B^{\prime}}
$$

then Razzaboni transformation of the surface coincides to the main Razzaboni surface. That is

$$
\varphi(\sigma)=\sigma
$$

In the case of $B=0$, which means that Bertrand geodesics of the surface $\sigma$ must have constant curvature, we have

$$
\kappa=\frac{1}{A}
$$

Thus, Razzaboni transformation is defined as

$$
\varphi(\sigma(u, v))=\sigma^{*}(u, v)=\sigma(u, v)+\frac{1}{\kappa} n(u, v)
$$


Since

$$
\kappa^{*}=-\frac{1}{A}=-\kappa
$$

then Razzaboni transformation maps the surface $\sigma$, whose Bertrand geodesics have constant curvature, to the surface $\sigma^{*}$, whose Bertrand geodesics also have constant curvature with the opposite sign. Moreover, the torsions of Bertrand geodesics of the surfaces satisfy the relation

$$
\tau \tau^{*}=\frac{1}{A^{2}}
$$

\section{REFERENCES}

[1]. Eisenhart L.P., A Treatise on the Differential Geometry of Curves and Surfaces. Dover, New York (1960).

[2]. Lopez R. Differential Geometry of Curves and Surfaces in Lorentz-Minkowski Space, arXiv:0810.3351v1, (2008).

[3]. Razzaboni A., Delle superficie nelle quali un sistema di geodetiche sono curve del Bertrand. Bologna Mem., 10 (1903) 539-548.

[4]. Schief W.K., On the Integrability of Bertrand curves and Razzaboni surfaces, Journal of Geometry and Physics, 45 (2002) 130-150.

[5]. Fibbi C., Sulle superficie che contengono un sistema di geodetiche a torsione constante, Pisa Ann., 5 (1888) 79-164.

[6]. Razzaboni A., Delle superficie nelle quali un sistema di geodetiche sono curve del Bertrand. Bologna, Tipografia Gamberini e Parmeggiani (1998).

[7]. Rogers C. and Schief W.K., Backlund and Darboux Transformations, Geometry of Modern Applications in Soliton Theory, Cambridge University Press, (2002).

[8]. Schief W.K. and Rogers C., Binormal Motion of Curves of Constant Curvature and Torsion, Generation of Soliton Surfaces, Proc. R. Soc. Lond. A., 455 (1999) 3163-3188.

[9]. Gürbüz N., The Motion of Timelike Surfaces in Timelike Geodesic Coordinates, Int. Journal of Math. Analysis, 4 (2010) 349-356.

[10]. Ding Q.and Inoguchi J., Schrödinger flows, binormal motion for curves and second AKNShierarchies, Chaos Solitons and Fractals, 21 (2004) 669-677.

[11]. Inoguchi J., Biharmonic curves in Minkowski 3-space, International Journal of Mathematics and Mathematical Sciences, 21 (2003) 1365-1368.

[12]. Erdoğdu M. and Özdemir M., Geometry of Hasimoto Surfaces in Minkowski 3-space. Mathematical Physics, Analysis and Geometry, 17 (2014) 169-281.

[13]. Inoguchi J. Timelike surfaces of constant mean curvature in Minkowski 3-space, Tokyo Journal of Mathematics, 21 (1998) 141-152.

[14]. Özdemir M. and Ergin A.A., Parallel Frames of Non-Lightlike Curves. Missouri Journal of Mathematical Sciences, 20 (2008) 127-137. 